\title{
Facile Synthesis of Nanostructured Anatase Titania with Controllable Morphology via Oxidation of TiC with Hydrogen Peroxide
}

\author{
Yuduo Ren and WenTao Hu \\ State Key Lab of Metastable Materials Science \& Technology, Yanshan University, Qinhuangdao 066004, China \\ Correspondence should be addressed to WenTao Hu; hwt@ysu.edu.cn
}

Received 1 April 2016; Revised 5 June 2016; Accepted 9 June 2016

Academic Editor: P. Davide Cozzoli

Copyright (C) 2016 Y. Ren and W. Hu. This is an open access article distributed under the Creative Commons Attribution License, which permits unrestricted use, distribution, and reproduction in any medium, provided the original work is properly cited.

Nanostructured anatase $\mathrm{TiO}_{2}$ with controllable morphology has been fabricated via the oxidation of TiC with $\mathrm{H}_{2} \mathrm{O}_{2}$. At room temperature, the reaction of TiC with $\mathrm{H}_{2} \mathrm{O}_{2}$ leads to dissolution of $\mathrm{TiC}$ into $\mathrm{H}_{2} \mathrm{O}_{2}$ aqueous solution, producing an acidic solution. By drying the acidic solution at $80^{\circ} \mathrm{C}$ in air, an amorphous powder of polytitanic acid with oxalate ligands is obtained, and its morphology is found to rely on the reaction time. By annealing the amorphous acidic powder at $T>350^{\circ} \mathrm{C}$, the nanostructured anatase $\mathrm{TiO}_{2}$ with controllable morphology is generated. Depending on the oxidation time, the morphology can be fabricated as sponge-like shape, flower-like shape, spongy balls, and so forth. The nanostructured anatase $\mathrm{TiO}_{2}$ is stable under the heating treatments until $900^{\circ} \mathrm{C}$, and its morphology can be tuned to the nanocrystalline grains. In addition to the annealing way, riceshaped anatase nanocrystals can be directly formed by aging the acidic solution under ambient conditions.

\section{Introduction}

Titania $\left(\mathrm{TiO}_{2}\right)$ is one of the mostly studied semiconductors. Since the discovery of the ability of $\mathrm{TiO}_{2}$ in splitting water [1], great interest has been aroused in nanostructured $\mathrm{TiO}_{2}$ owing to the various applications in solar energy conversion and photodegradation of organic pollutants $[2,3]$. In those applications, one of the important parameters is the specific surface area, which is strongly related to the nanostructured morphology. To date, many synthetic routes have been developed to prepare the nanostructured $\mathrm{TiO}_{2}$ with different morphologies, for example, fabrication of $\mathrm{TiO}_{2}$ nanoparticles with sol-gel method $[4,5]$, successful syntheses of $\mathrm{TiO}_{2}$ nanowires $[6,7]$, nanorods $[8,9]$, and nanotubes $[10,11]$ with hydrothermal method, and preparation of $\mathrm{TiO}_{2}$ nanowires $[12,13]$, thin film [14], and nanorods $[15,16]$ with solvothermal method. In these synthetic routes, however, the subsequent thermal treatment is usually required for crystallization and removal of residual contaminants. Recently, direct oxidation of pure titanium has been reported for the preparation of $\mathrm{TiO}_{2}$ nanomaterials. By inserting a cleaned Ti plate into hydrogen peroxide solution, $\mathrm{TiO}_{2}$ nanorods can be formed through the oxidation of Ti with $\mathrm{H}_{2} \mathrm{O}_{2}$ at low temperature $[17,18]$. By using acetone as the oxygen source, the oxidation of $\mathrm{Ti}$ with acetone at high temperature is also able to produce $\mathrm{TiO}_{2}$ nanorods [19]. It has been reported that $\mathrm{TiO}_{2}$ nanotubes can be fabricated by anodic oxidation of a Ti foil [20].

Similar to Ti, TiC can be also easily dissolved into $\mathrm{H}_{2} \mathrm{O}_{2}$ aqueous solution at room temperature via the oxidation with $\mathrm{H}_{2} \mathrm{O}_{2}$, producing an acidic solution. By drying the acidic solution, an amorphous powder of polytitanic acid with oxalate ligands is able to be obtained as precursor for fabrication of nanostructured anatase $\mathrm{TiO}_{2}[21,22]$. In the present work, detailed investigations including XRD, Raman [23], SEM, and TEM have been performed on fabrication of nanostructured anatase $\mathrm{TiO}_{2}$ via the oxidation of TiC with $\mathrm{H}_{2} \mathrm{O}_{2}$.

\section{Experimental}

2.1. Synthesis. Commercial TiC powders (99.5\% purity, $3 \mu \mathrm{m}$ in average size) and $30 \% \mathrm{H}_{2} \mathrm{O}_{2}$ aqueous solution were used as the original materials in the syntheses of nanostructured anatase $\mathrm{TiO}_{2}$. TiC powders were dissolved into $\mathrm{H}_{2} \mathrm{O}_{2}$ aqueous 
solution to produce an acidic solution. By drying the acidic solution in air at $80^{\circ} \mathrm{C}$, amorphous powders of polytitanic acid with oxalate ligands were then obtained. Nanostructured anatase $\mathrm{TiO}_{2}$ was fabricated by annealing the amorphous acidic powders in a homemade tubular furnace at high temperatures.

2.2. Characterization. $\mathrm{X}$-ray diffraction (XRD) patterns were collected using XRD diffractometer (Smartlab Rigaku, Japan) with $\mathrm{Cu} \mathrm{Ka}$ radiation of $l=1.54056 \AA$. Raman scattering was performed in a Raman spectrometer (Renishaw, New Mills, UK) with an Ar laser (514.5 nm). The infrared pattern was measured with the FTIR-8100 FT-IR spectrometer (Shimadzu, Kyoto, Japan). The morphologies of the samples were checked in a scanning electron microscopy (S4800, Hitachi, Japan) and transmission electron microscopy (JEM-2010, JEOL, Japan). In the TEM and HRTEM observations, the samples were prepared as follows: a small amount of the fabricated powders dispersed in ethanol. After the ultrasonic vibration for $15 \mathrm{~min}$, a drop of the dispersion was placed onto a copper grid coated with a layer of amorphous carbon.

\section{Results and Discussion}

3.1. Oxidation of $\mathrm{TiC}$ with $\mathrm{H}_{2} \mathrm{O}_{2}$. TiC powders can be dissolved in colorless $\mathrm{H}_{2} \mathrm{O}_{2}$ aqueous solution at room temperature via the oxidation of $\mathrm{TiC}$ with $\mathrm{H}_{2} \mathrm{O}_{2}$, and a solution of polytitanic acid with oxalate ligands is produced [22]. In the dissolution process, a great deal of gas is released via serious bubbles and the solution color changes quickly to orange (see inset of Figure S1 in supporting information). The monitoring of IR spectroscopy indicates that the released gas is dominantly $\mathrm{CO}_{2}$ (see Figure $\mathrm{S} 1$ in supporting information, available online at http://dx.doi.org/10.1155/2016/6325615). It is observed that the color of acidic solution depends on the reaction time. By maintaining the reaction, the acidic solution changes gradually to yellow in color (see Figure S2 in supporting information), and a yellow gel can be finally obtained. By drying the acidic solution or gel at $80^{\circ} \mathrm{C}$ in air, amorphous powder can be obtained, and its color relies on the oxidation duration (see Figure S2 in supporting information). The infrared spectrum of the amorphous powder (see Figure S3 in supporting information) is found to be similar to the previously reported ones of polytitanic acid with oxalate ligands [21, 22].

In the present work, the nanostructured anatase $\mathrm{TiO}_{2}$ was fabricated by annealing the amorphous powders of polytitanic acid with oxalate ligands at high temperatures. To investigate the influence of oxidation time on the morphology of nanostructured anatase $\mathrm{TiO}_{2}$, the dissolution of TiC into $\mathrm{H}_{2} \mathrm{O}_{2}$ aqueous solution was performed in a series of glass beakers (marked with first reaction time of $3 \mathrm{~h}, 10 \mathrm{~h}, 14 \mathrm{~h}$, $20 \mathrm{~h}$, and $7 \mathrm{~h}$ ) for different durations. When the desired time was over, the residual $\mathrm{TiC}$ particles and/or any insoluble impurities were removed out. The amorphous powders of polytitanic acid with oxalate ligands were then obtained by drying the acidic solution or gel in air at $80^{\circ} \mathrm{C}$. The amorphous acidic powders were finally annealed in a homemade tubular furnace at high temperatures in air to fabricate nanostructured anatase $\mathrm{TiO}_{2}$.

3.2. Nanostructured Anatase $\mathrm{TiO}_{2}$ with Controllable Morphology. When the reaction was kept for 3 hours, a little amount of the supernatant or acidic solution was abstracted to check the oxidation product via the TEM measurements. As shown in the TEM image of Figure 1(a), the oxidation of TiC with $\mathrm{H}_{2} \mathrm{O}_{2}$ leads to the formation of spherical nanoparticles. After the reaction was maintained for 7 hours, the residual TiC particles were filtered out. The obtained supernatant was checked with TEM. As shown in the TEM image of Figure 1(b), the oxidation-produced nanoparticles become smaller in size and deviate from the spherical shape, but they are connected with each other to form an irregular network. The collected SAED pattern (the left inset in Figure 1(b)) indicates that the nanoparticles are in the amorphous state. The supernatant was firstly dried at $80^{\circ} \mathrm{C}$. The XRD pattern of dried powders (right inset in Figure 1(b)) confirms the amorphous state. By annealing the dried powders at $500^{\circ} \mathrm{C}$ in air for one hour, the TEM image in Figure 1(c) shows that the connected nanoparticles ( $20 \mathrm{~nm}$ in average size) become more regular in shape. The XRD pattern (inset of Figure 1(c)) displays that the annealing leads to the formation of anatase phase. Figure 1(d) gives the HRTEM image for one of the nanoparticles, revealing the good crystallinity. The corresponding fast Fourier transform (FFT) power pattern (inset in Figure 1(d)) supports that the annealed nanoparticles are in anatase phase.

After 10-hour reaction of $\mathrm{TiC}$ with $\mathrm{H}_{2} \mathrm{O}_{2}$, the residual TiC particles were then removed, and the filtered-out supernatant was observed by the TEM measurements. As shown in Figure 2(a), the TEM images indicate that the oxidation product evolved into a sponge-like porous structure. It can be recognized that the sponge-like structure is formed by entwining $2 \mathrm{D}$ nanosheets. In Figure 2(b), the HRTEM image of a nanosheet displays distorted atomic fringes, and consistently the weak diffraction rings can be recognized in the SAED of inset. Therefore, the nanosheets are crystallized to a certain extent. After drying the supernatant at $80^{\circ} \mathrm{C}$, the obtained powder was characterized by XRD. In inset on the top right corner of Figure 2(a), the XRD pattern demonstrates the appearance of several broad reflection peaks, confirming the observations of HRTEM and SAED measurements. The dried powder was annealed at $350^{\circ} \mathrm{C}$ in air for 2 hours. The TEM image in Figure 2(c) displays that the annealed product is highly porous, similar to a sponge in shape. In Figure 2(d), the XRD and Raman scattering characterizations reveal that the annealing induces the formation of anatase phase.

When the oxidation of TiC with $\mathrm{H}_{2} \mathrm{O}_{2}$ was kept longer than 10 hours, precipitates were observed to appear gradually. The oxidation was maintained for 14 hours; the residual TiC particles were then removed. As shown in the TEM image of Figure 3(a), the oxidation product was developed into urchin-like particles in the microscale. Clearly, these urchinlike particles are formed by aggregations of $2 \mathrm{D}$ nanosheets. 




(a)

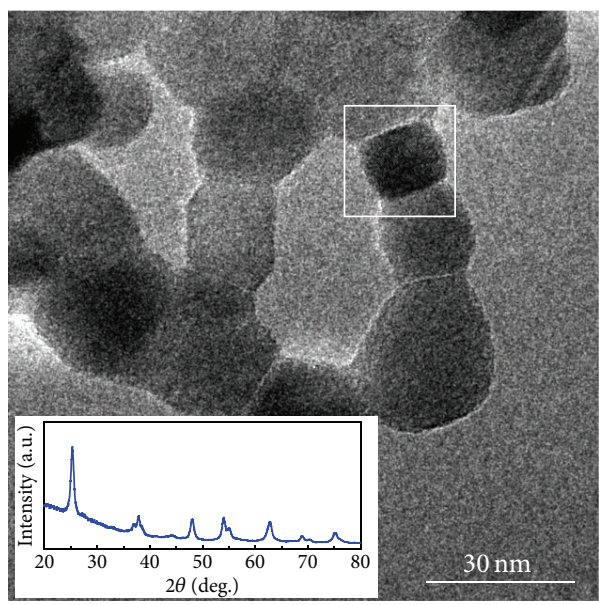

(c)



(b)

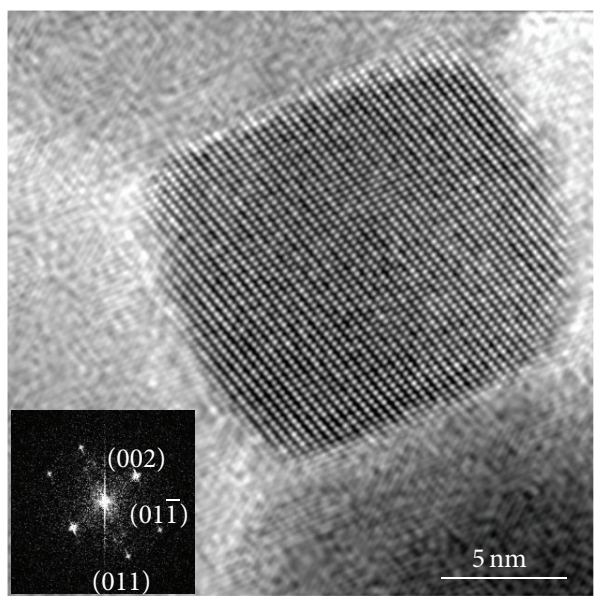

(d)

FIGURE 1: (a) TEM image for a powder of polytitanic acid with oxalate ligands obtained after the oxidation of TiC with $\mathrm{H}_{2} \mathrm{O}_{2}$ for 3 hours. (b) TEM image for a powder of polytitanic acid with oxalate ligands obtained after the oxidation of TiC with $\mathrm{H}_{2} \mathrm{O}_{2}$ for 7 hours. Inset is the SAED pattern, showing amorphous state of the acidic powder. (c) TEM image and RT XRD pattern (inset) for the nanostructured anatase $\mathrm{TiO}_{2}$ obtained by annealing the amorphous acidic powder in (b) at $500^{\circ} \mathrm{C}$ for 1 hour. (d) HRTEM image for one of the anatase nanocrystals in (c). Inset is the corresponding FFT of the HRTEM image, in which the bright points can be well indexed to the anatase structure.

In the collected SAED in inset of Figure 3(a), clear diffraction rings can be observed. The HRTEM image of a nanosheet in Figure 3(b) also reveals the presence of well crystallized grains, being consistent with the SAED observation. After drying at $80^{\circ} \mathrm{C}$ in air, however, the $\mathrm{XRD}$ pattern of the dried powder is found to be similar to the one in inset of Figure 2(b). The dried powder was annealed in air at $700^{\circ} \mathrm{C}$ for 2 hours. As shown in Figure 3(c), the TEM image indicates that the annealed powder exhibits a flower-like shape. As demonstrated in Figure 3(d), the XRD and Raman scattering pattern reveal the annealing-induced formation of anatase phase.

When the oxidation reaction was kept longer than 14 hours, more precipitates were observed to appear. After the reaction of 20 hours, the residual $\mathrm{TiC}$ particles were filtered out, and the oxidation product was checked by the TEM measurements. The TEM image in Figure 4(a) shows that the oxidation product exhibits the morphology of fluffy balls. The fluffy balls can be imagined to originate from aggregations of $2 \mathrm{D}$ nanosheets in a huge amount. In the collected SAED of inset in Figure 4(a), diffraction rings are clearly observed, and some diffraction spots are also recognized. In the amplified TEM image in Figure 4(b), many grains are observed inside the nanosheets, and the HRTEM image of one grain in inset shows the good crystallinity. After drying in air at $80^{\circ} \mathrm{C}$, however, the obtained powder still produces the XRD pattern similar to the previous ones in inset of Figure 2(b). At $750^{\circ} \mathrm{C}$ in air, the annealing was performed on the dried powder for 2 hours. As shown in the SEM image of Figure 4(c) and the enlarged one in inset, the annealed product is found to 




(a)

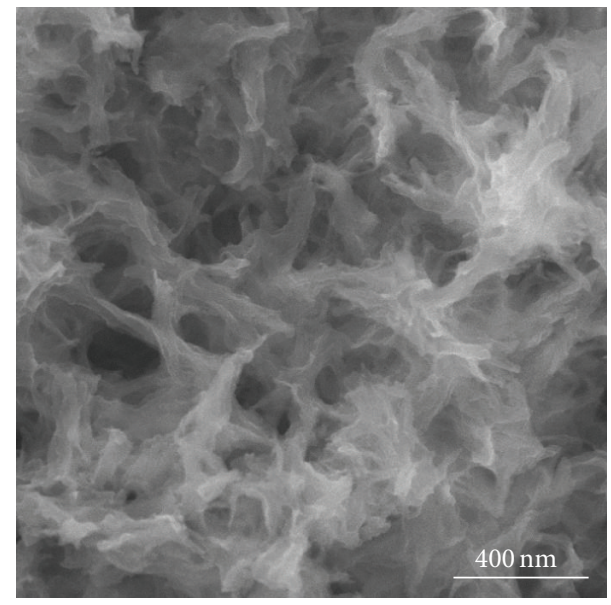

(c)



(b)
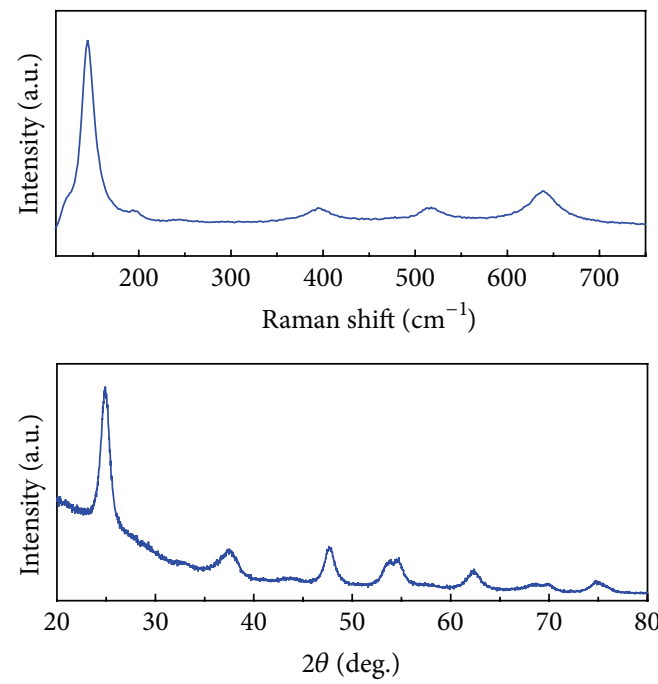

(d)

FIGURE 2: (a) TEM image for the acidic powder obtained after the oxidation of TiC with $\mathrm{H}_{2} \mathrm{O}_{2}$ for 10 hours, displaying the meshed network of entwining 2D nanosheets. (b) HRTEM image of a 2D nanosheet, demonstrating the observable atomic fringes. Inset on the left lower corner is the SAED, showing the diffraction rings. Inset on the right top corner is the RT XRD of acidic powder in (a), demonstrating the amorphous state. (c) SEM image for the nanostructured anatase $\mathrm{TiO}_{2}$ obtained by annealing the amorphous acidic powder in (b) at $350^{\circ} \mathrm{C}$ for 2 hours. (d) RT Raman (top) and XRD pattern (bottom) for the annealing-produced anatase $\mathrm{TiO}_{2}$ in (d).

become highly porous microspheres. The XRD and Raman pattern in Figure 4(d) are indicative of the annealing-induced formation of anatase phase.

The oxidation of TiC with $\mathrm{H}_{2} \mathrm{O}_{2}$ was maintained for 7 hours, same as the reaction of $10 \mathrm{~h}$. By removing the residual TiC particles, however, the orange supernatant or acidic solution was kept for aging under the ambient conditions. By aging for 3 days, the orange supernatant was transformed to opaque yellow gel. The TEM observations of the gel in Figure 5(a) reveal the morphology of cottony hairs. Inside the cottony hairs, the TEM image in inset of Figure 5(a) is indicative of the presence of many nanocrystals with the average size less than $2 \mathrm{~nm}$. The further aging leads to the gradual appearance of precipitates. By aging for 7 days, the precipitates became white in color (see Figure S4 in supporting information). Figure 5(b) shows the TEM image of the precipitates after the aging for 7 days. On a network background made up of the cottony hairs, many rice-shaped nanoparticles are observed to be present. Inset of Figure 5(b) shows the TEM image of a rice-shaped nanoparticle. On the top end, aggregation of some cottony hairs is still observable, indicating that the nanoparticle is not completely well developed. Moreover, as marked out by red ovals in Figure 5(b), it can be recognized that the aggregations of cottony hairs tend to evolve into the riceshaped nanoparticles. Thereby, the rice-shaped nanoparticles should evolve from aggregation of the cottony hairs. On the TEM image (inset of Figure 5(b)), it can be seen clearly 


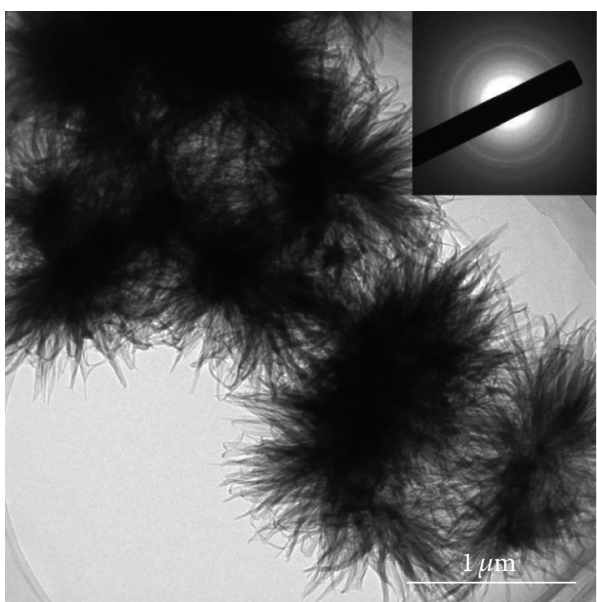

(a)

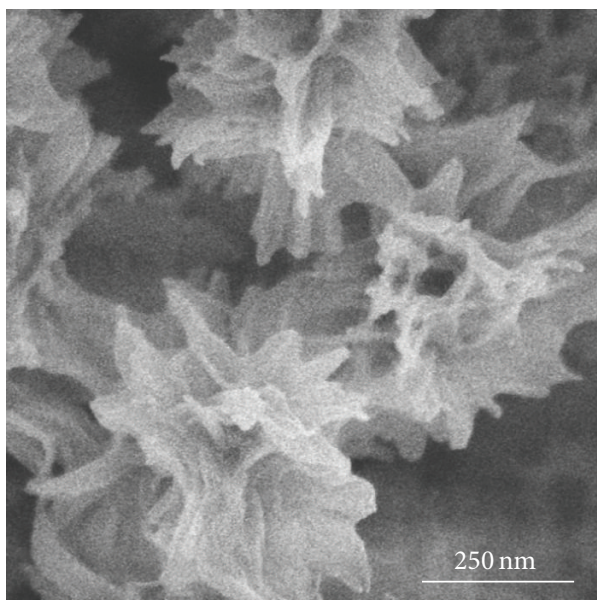

(c)

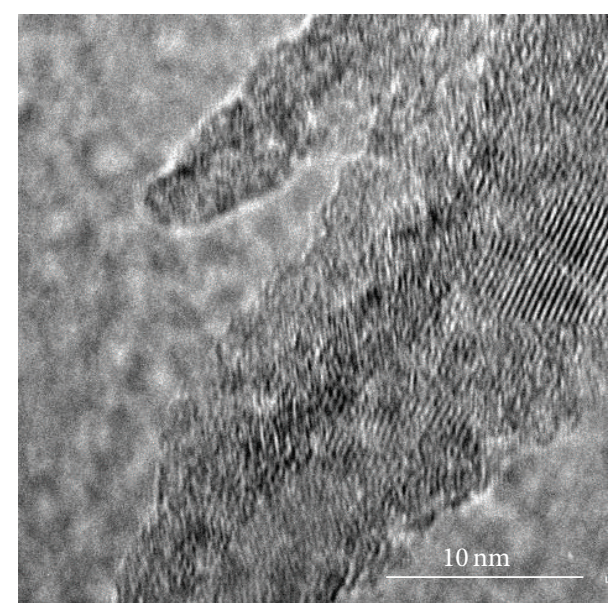

(b)
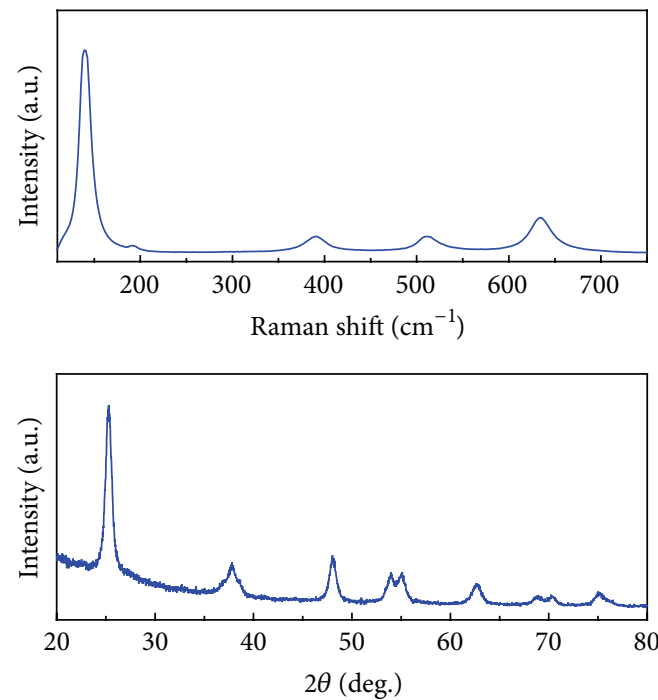

(d)

FIgURE 3: (a) TEM image for the acidic powder obtained after the oxidation of TiC with $\mathrm{H}_{2} \mathrm{O}_{2}$ for 14 hours, displaying the urchin-like morphology made of entwining 2D nanosheets. Inset is the SAED showing the observable diffraction rings. (b) HRTEM image of a 2D nanosheet, demonstrating the local good crystallization. (c) SEM image for the nanostructured anatase $\mathrm{TiO}_{2}$ obtained by annealing the amorphous acidic powder in (b) at $700^{\circ} \mathrm{C}$ for 2 hours, showing the flower-like morphology. (d) RT Raman (top) and XRD pattern (bottom) for the annealing-produced anatase $\mathrm{TiO}_{2}$ in (c).

that the rice-shaped nanoparticle is actually constructed with many nanocrystals with the average size less than $5 \mathrm{~nm}$. Figure 5(c) is the HRTEM image of the rice-shaped nanoparticle, and inset is the corresponding FFT pattern. It can be identified that the nanocrystals are assembled with smaller mosaic blocks, which arranged almost along the same orientation with only a small deviation. The presence of a small deviation in orientation leads to the splitting of bright spots on the FFT pattern. As marked out with the red circles, the presence of nanopores can be recognized between the nanocrystals, which formed owing to the deviations between those smaller mosaic blocks. Based on the HRTEM image and the corresponding FFT pattern, it can be determined that the rice-shaped nanoparticle has the anatase structure.

As shown in the TEM image of Figure 6(a), the aging for two months leads to the complete formation of rice-shaped nanoparticles. The good crystallinity of the nanoparticles is displayed by the sharp rings in inset of Figure 6(a). The Raman and XRD patterns in Figure 6(b) indicate the anatase phase of the nanoparticles. Figure 6(c) shows the HRTEM image of rice-shaped nanoparticles and the corresponding FFT pattern (inset). It can be determined that the nanoparticle is well crystallized along the [100] zone axis. As shown in the enlarged HRTEM image of Figure 6(d), the presence of 




(a)



(c)

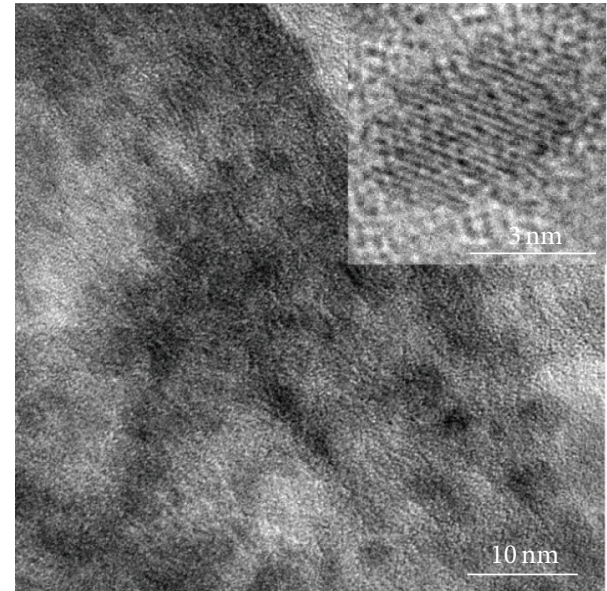

(b)
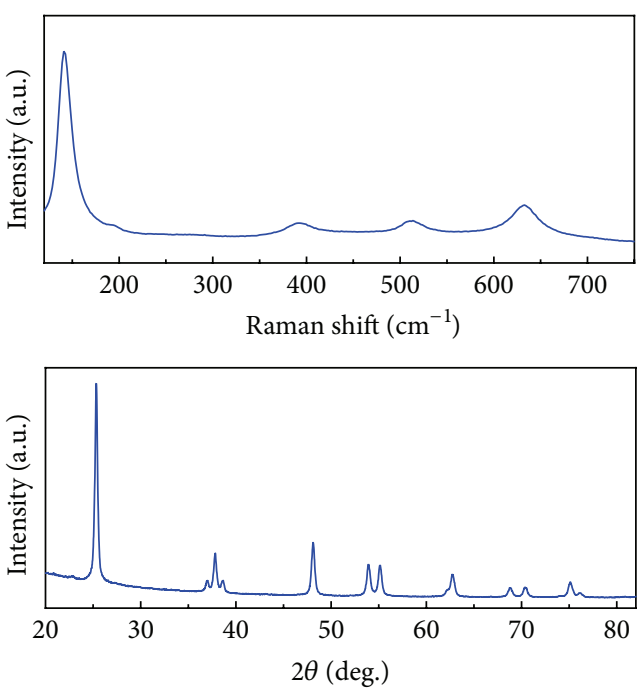

(d)

Figure 4: (a) TEM image for the acidic powder obtained after the oxidation of TiC with $\mathrm{H}_{2} \mathrm{O}_{2}$ for 20 hours, displaying the morphology of spongy balls made of entwining 2D nanosheets. Inset is the SAED showing the observable diffraction rings. (b) HRTEM image of a 2D nanosheet, demonstrating the presence of crystallized nanograins. (c) SEM image for the nanostructured anatase $\mathrm{TiO}_{2}$ obtained by annealing the amorphous acidic powder in (b) at $750^{\circ} \mathrm{C}$ for 2 hours, showing the morphology of spongy spheres. Inset is an enlarged SEM image for the surface of a spongy sphere. (d) RT Raman (top) and XRD pattern (bottom) for the annealing-produced anatase $\mathrm{TiO}_{2}$ in (c).

nanopores is still observable in the rice-shaped nanoparticle, indicating that it evolves from the one constructed with the smaller nanocrystals.

\subsection{Annealing Effect on Nanostructured Anatase $\mathrm{TiO}_{2}$. After} 10-hour reaction of TiC with $\mathrm{H}_{2} \mathrm{O}_{2}$, the amorphous powder of polytitanic acid with oxalate ligands was obtained. As shown in Figures 2(c) and 2(d), nanostructured anatase $\mathrm{TiO}_{2}$ with a sponge-like shape was fabricated by annealing of the amorphous acidic powder in air at $350^{\circ} \mathrm{C}$ for two hours. Figure 7(a) shows the XRD pattern for the nanostructured anatase $\mathrm{TiO}_{2}$ obtained by annealing the amorphous acidic powder at different temperatures above $350^{\circ} \mathrm{C}$ for two hours. Even after the annealing at $900^{\circ} \mathrm{C}$, the obtained
$\mathrm{TiO}_{2}$ is dominantly in the anatase phase, indicating that the fabricated anatase $\mathrm{TiO}_{2}$ is stable until $900^{\circ} \mathrm{C}$. Moreover, the reflection peaks are shifted owing to the annealing at different temperatures, but the shift directions of peaks are not consistent. As shown clearly in inset of Figure 7(a), some reflection peaks are shifted consistently towards the low angle with the increase of annealing temperature, while the others are shifted firstly towards the high angle and then towards the low angle. The lattice parameters are determined from the XRD patterns and are shown in Figure 7(b). With the rise of annealing temperature, the lattice parameter $c$ exhibits a monoclinic increase, while for the lattice parameter $a$ or $b$, a quick decrease occurs firstly below $\sim 700^{\circ} \mathrm{C}$ and then a slow increase appears above $\sim 700^{\circ} \mathrm{C}$. The volume of unit cell was 


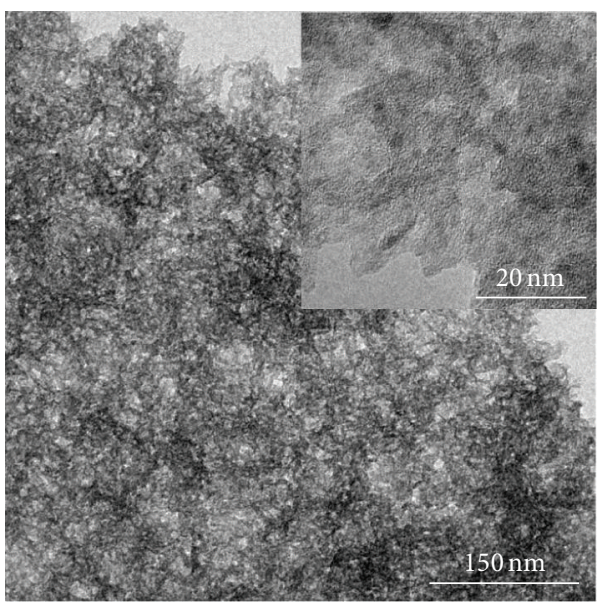

(a)

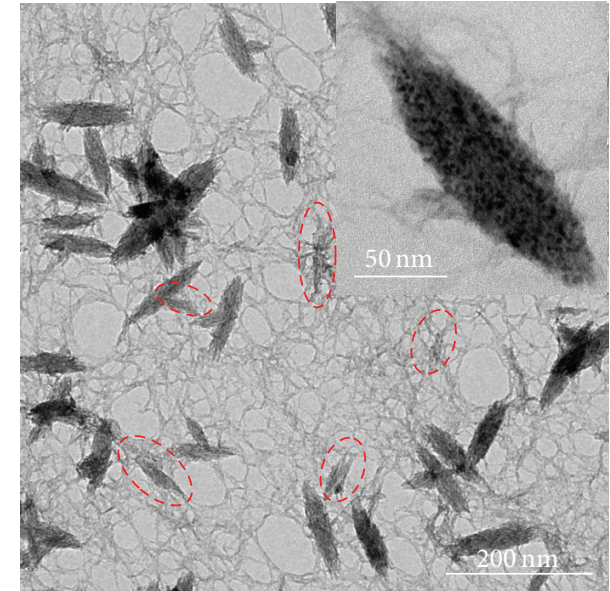

(b)

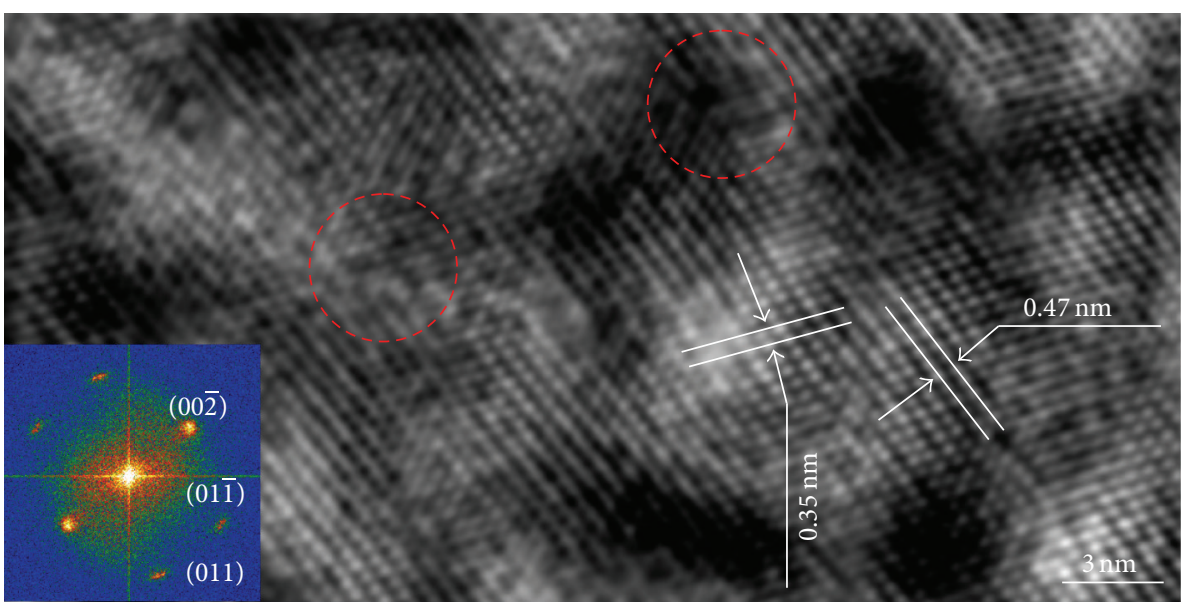

(c)

FIgURE 5: The aging effect on the acidic solution produced by dissolution of TiC into $\mathrm{H}_{2} \mathrm{O}_{2}$ aqueous solution for 10 hours. (a) TEM image showing that the meshed network of entwining 2D nanosheets was changed to the meshed network of cottony hairs by the aging for 3 days. Inset is an enlarged TEM image showing the presence of many crystallized nanograins. (b) TEM image showing the formation of riceshaped nanoparticles after the aging for 7 days. On the meshed background, some undeveloped nanoparticles are marked out by red ovals, indicating that the rice-shaped nanoparticles should evolve from aggregation of cottony hairs. Inset is an enlarged TEM image of a rice-shaped nanoparticle, revealing that the rice-shaped nanoparticle is actually constructed with many nanocrystals of size less than $5 \mathrm{~nm}$. (c) HRTEM image of the rice-shaped nanoparticle (inset in (b)) and the corresponding FFT power pattern (inset). The red circles label out the nanopores.

calculated and shown as inset in Figure 7(b). Obviously, the unit cell is induced to shrink with the increase of annealing temperature below $700^{\circ} \mathrm{C}$ but expand above $700^{\circ} \mathrm{C}$.

As shown in Figure $7(\mathrm{a})$, the reflection peak width becomes narrower with the increase of annealing temperatures, being suggestive of the annealing-induced increase in the grain size. The average grain sizes were calculated from the most intense refection peak (110) using the Scherrer formula of $D=0.9 \lambda / \beta \cos \theta$, where $\lambda$ is the $\mathrm{X}$-ray wavelength, $\theta$ is the diffraction angle of the (110) peak, and $\beta$ is the halfwidth after subtracting the instrumental broadening. Inset of Figure 7(b) demonstrates the variation of $D$ with the annealing temperature. The rise of annealing temperature leads to the monoclinic increase in the average grain size.
Even after the heating treatment at $900^{\circ} \mathrm{C}$ for 2 hours, the average grain size for the dominant anatase $\mathrm{TiO}_{2}$ is still in the nanoscale. The annealing-induced change in morphology was checked by the SEM measurements. Figure 8 shows the SEM images obtained after the heating treatments at four typical temperatures. As shown in Figure 8(a), after the annealing at temperatures below $700^{\circ} \mathrm{C}$, the sponge-like morphology is maintained for the obtained nanostructured anatase $\mathrm{TiO}_{2}$, but the pores become smaller with the rise of annealing temperature. After the annealing at $700^{\circ} \mathrm{C}$, Figure 8(b) reveals that the nanostructured morphology is still similar to the sponge-like ones, but nanograins can be recognized to appear. When the annealing was performed at $800^{\circ} \mathrm{C}$ and above, Figures $8(\mathrm{c})$ and $8(\mathrm{~d})$ reveal clearly the 




(a)

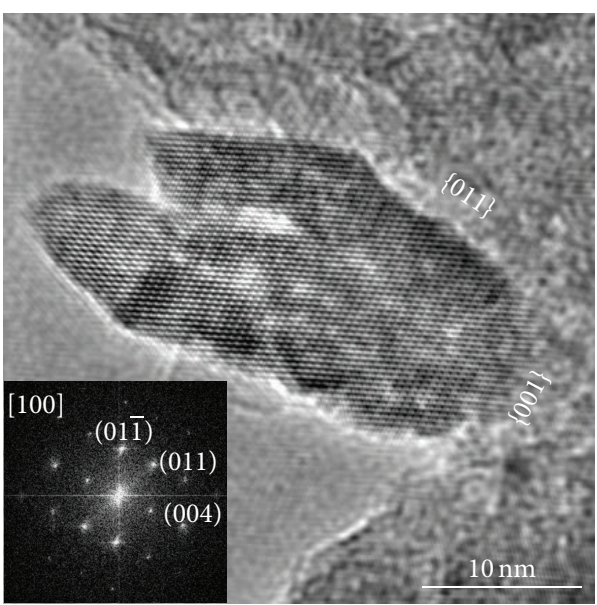

(c)
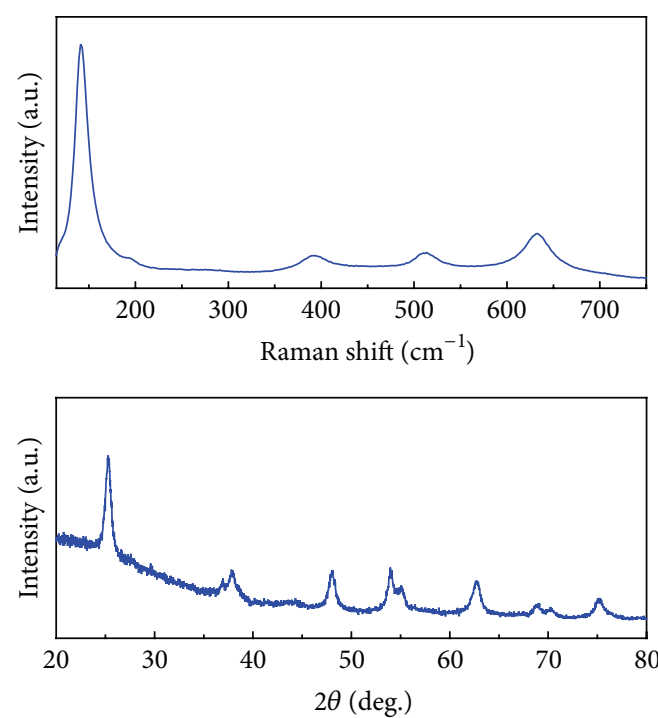

(b)

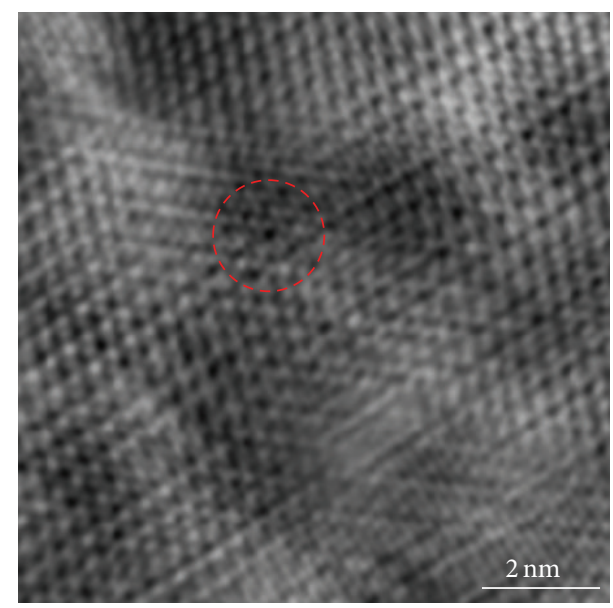

(d)

FIGURE 6: (a) The well-developed rice-shaped nanoparticles obtained after the aging for 2 months. Inset is the SAED pattern. (b) RT Raman and XRD pattern for the rice-shaped nanoparticles in (a), being indicative of the anatase phase. (c) HRTEM image of a rice-shaped nanoparticle and the corresponding FFT power pattern, indicating the good crystallinity of ice-shaped nanoparticles. (d) The enlarged HRTEM image showing the presence of nanopores as marked out by the red circle.

formation of nanocrystalline grains. This annealing-induced change in nanostructured morphology could be considered as the origin for the abnormal variations of the lattice parameters and unit cell volume.

3.4. Conclusions. TiC can be easily dissolved into $\mathrm{H}_{2} \mathrm{O}_{2}$ aqueous solution at room temperature via the oxidation with $\mathrm{H}_{2} \mathrm{O}_{2}$, producing an acidic solution. By drying the acidic solution at $80^{\circ} \mathrm{C}$ in air, an amorphous powder of polytitanic acid with oxalate ligands is obtained as precursor for fabrication of nanostructured anatase $\mathrm{TiO}_{2}$. It is observed that morphology of the prepared amorphous acidic powder depends on the oxidation time. Relying on the oxidation time, annealing of the obtained amorphous acidic powder is able to produce the nanostructured anatase $\mathrm{TiO}_{2}$ with controllable morphology. By heating the nanostructured anatase $\mathrm{TiO}_{2}$ with a sponge-like morphology, it is found that the anatase phase is stable until $900^{\circ} \mathrm{C}$, and the morphology is changed to the nanocrystalline grains. In addition to the annealing way, it is found that rice-shaped anatase nanocrystals can be directly formed by aging the acidic solution under the ambient conditions.

\section{Additional Points}

(i) Hydrogen peroxide is used as the oxidant, producing no environmental contaminations. (ii) By controlling the reaction time of TiC with $\mathrm{H}_{2} \mathrm{O}_{2}$, the morphology of the produced nanostructured anatase $\mathrm{TiO}_{2}$ can be easily tuned. (iii) Aging 


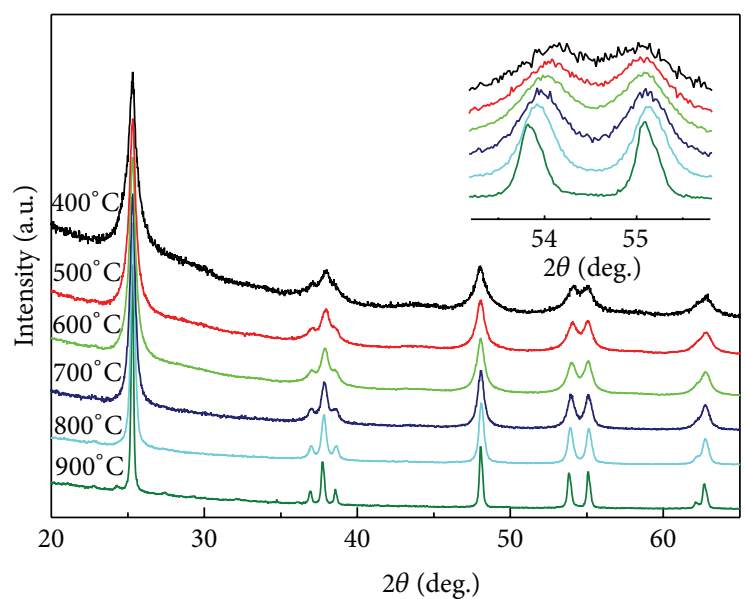

(a)
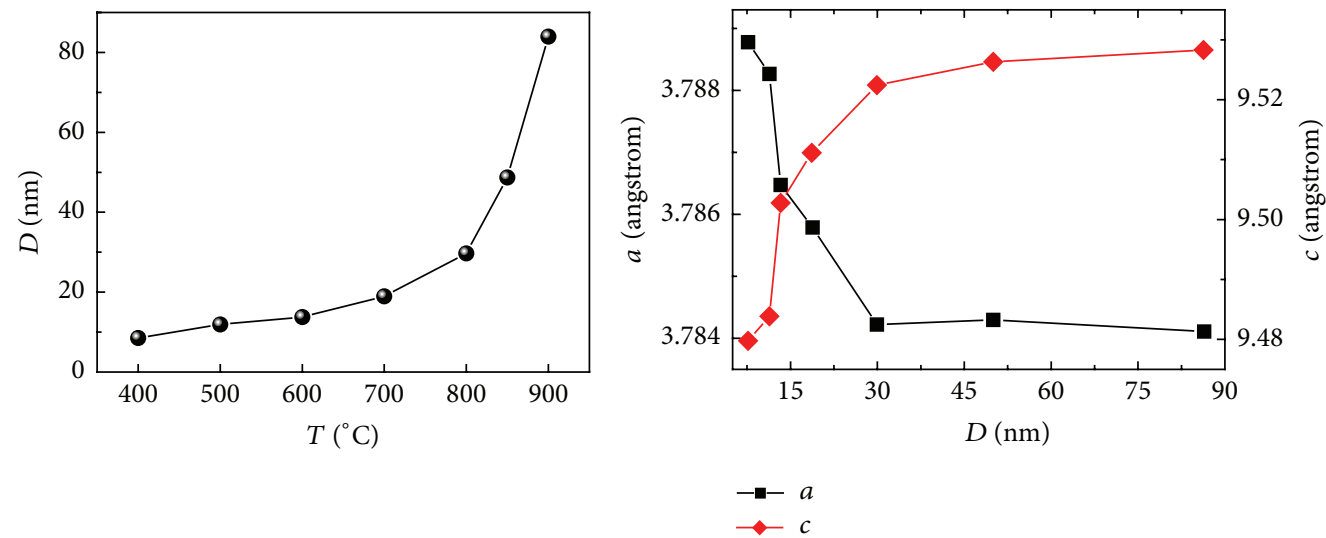

(A)

(B)
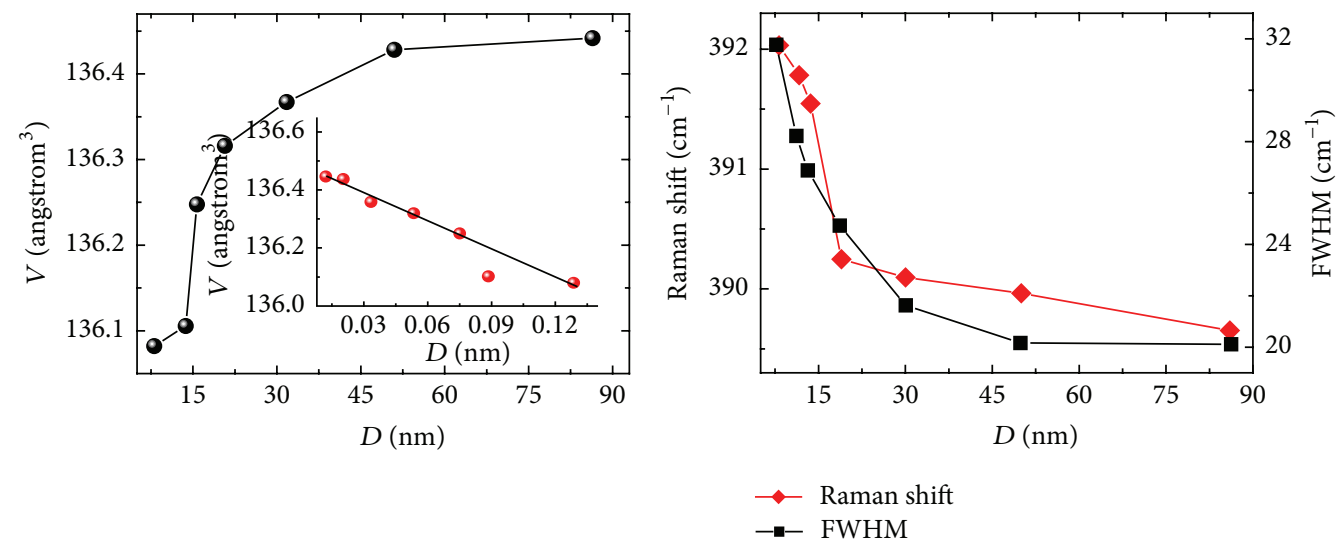

(C)

(D)

(b)

FIGURE 7: (a) The XRD pattern for the nanostructured $\mathrm{TiO}_{2}$ after the annealing at series of temperatures for 2 hours. Inset is the amplified peaks around $2 \theta=54$ and $55^{\circ}$. (b) The variations of lattice parameters, unit cell volume, and average grain size with the annealing temperature.

at room temperature is able to induce the crystallization of nanostructured anatase $\mathrm{TiO}_{2}$, thus favorable for energy conservation.

\section{Competing Interests}

The authors have declared that no competing interests exist. 


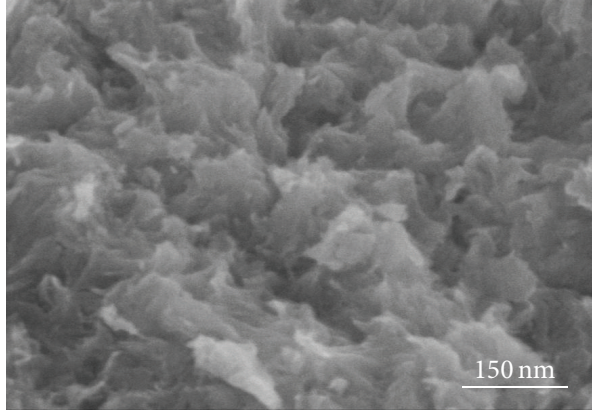

(a)

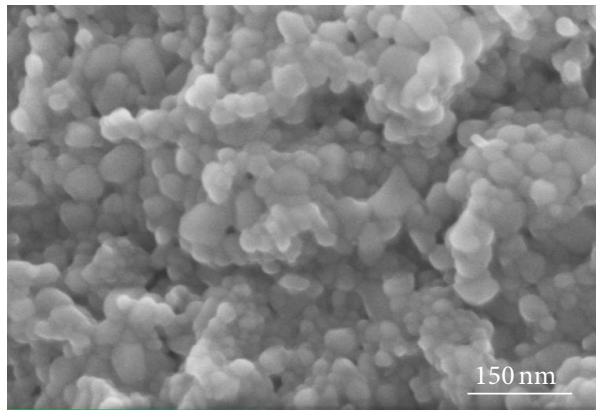

(c)

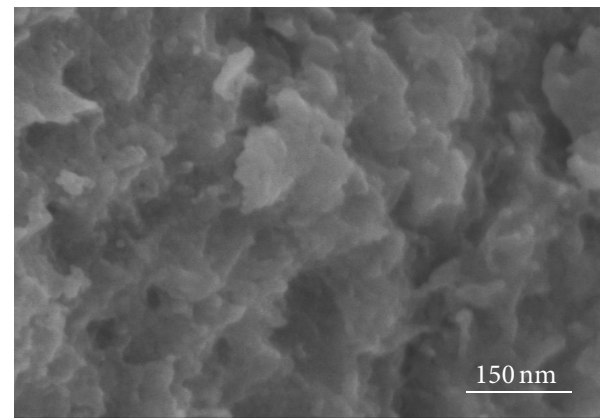

(b)

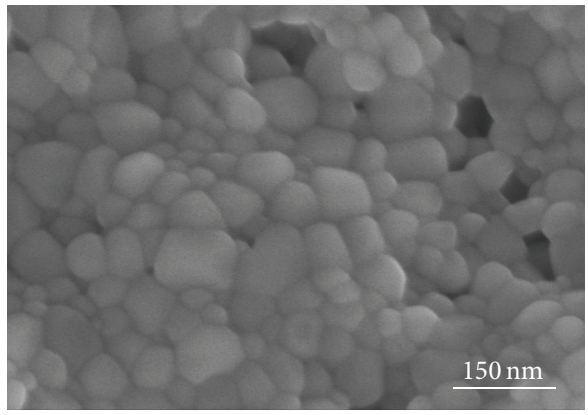

(d)

FIGURE 8: SEM images of the nanostructured anatase $\mathrm{TiO}_{2}$ after the annealing at 500 (a), 700 (b), 800 (c), and $900^{\circ} \mathrm{C}(\mathrm{d})$.

\section{Acknowledgments}

The authors would like to thank the financial supports from the National Basic Research Program of China (Grant no. 511CB808205) and Natural Science Foundations of China (Grant nos. 51272225, 51121061, and 51102206).

\section{References}

[1] N. Serpone, D. Lawless, and R. Khairutdinov, "Size effects on the photophysical properties of colloidal anatase $\mathrm{TiO}_{2}$ particles: size quantization or direct transitions in this indirect semiconductor?" The Journal of Physical Chemistry, vol. 99, no. 45, pp. 16646-16654, 1995.

[2] X. Chen and S. S. Mao, "Titanium dioxide nanomaterials: synthesis, properties, modifications and applications," Chemical Reviews, vol. 107, no. 7, pp. 2891-2959, 2007.

[3] A. Kubacka, M. Fernández-García, and G. Colón, "Advanced nanoarchitectures for solar photocatalytic applications," Chemical Reviews, vol. 112, no. 3, pp. 1555-1614, 2012.

[4] A. Chemseddine and T. Moritz, "Nanostructuring titania: control over nanocrystal structure, size, shape, and organization," European Journal of Inorganic Chemistry, no. 2, pp. 235-245, 1999.

[5] T. Sugimoto, X. Zhou, and A. Muramatsu, "Synthesis of uniform anatase $\mathrm{TiO}_{2}$ nanoparticles by gel-sol method: 4 . Shape control," Journal of Colloid and Interface Science, vol. 259, no. 1, pp. 53-61, 2003.

[6] A. R. Armstrong, G. Armstrong, J. Canales, R. García, and P. G. Bruce, "Lithium-ion intercalation into $\mathrm{TiO}_{2}-\mathrm{B}$ nanowires," Advanced Materials, vol. 17, no. 7, pp. 862-865, 2005.
[7] A. R. Armstrong, G. Armstrong, J. Canales, and P. G. Bruce, " $\mathrm{TiO}_{2}-\mathrm{B}$ nanowires", Angewandte Chemie-International Edition, vol. 43, no. 17, pp. 2286-2288, 2004.

[8] Q. Zhang and L. Gao, "Preparation of oxide nanocrystals with tunable morphologies by the moderate hydrothermal method: insights from rutile $\mathrm{TiO}_{2}$," Langmuir, vol. 19, no. 3, pp. 967-971, 2003.

[9] X. Feng, J. Zhai, and L. Jiang, "The fabrication and switchable superhydrophobicity of $\mathrm{TiO}_{2}$ nanorod films," Angewandte Chemie International Edition, vol. 44, no. 32, pp. 5115-5118, 2005.

[10] G. Gundiah, S. Mukhopadhyay, U. G. Tumkurkar, A. Govindaraj, U. Maitra, and C. N. R. Rao, "Hydrogel route to nanotubes of metal oxides and sulfates," Journal of Materials Chemistry, vol. 13, no. 9, pp. 2118-2122, 2003.

[11] T. Kasuga, M. Hiramatsu, A. Hoson, T. Sekino, and K. Niihara, "Titania nanotubes prepared by chemical processing," Advanced Materials, vol. 11, no. 15, pp. 1307-1311, 1999.

[12] B.-M. Wen, C.-Y. Liu, and Y. Liu, "Solvothermal synthesis of ultralong single-crystalline $\mathrm{TiO}_{2}$ nanowires," New Journal of Chemistry, vol. 29, no. 7, pp. 969-971, 2005.

[13] B. Wen, C. Liu, and Y. Liu, "Bamboo-shaped Ag-Doped $\mathrm{TiO}_{2}$ nanowires with heterojunctions," Inorganic Chemistry, vol. 44, no. 19, pp. 6503-6505, 2005.

[14] Z. Tebby, O. Babot, T. Toupance, D.-H. Park, G. Campet, and M.-H. Delville, "Low-temperature UV-processing of nanocrystalline nanoporous Thin $\mathrm{TiO}_{2}$ films: an original route toward plastic electrochromic systems," Chemistry of Materials, vol. 20, no. 23, pp. 7260-7267, 2008.

[15] X. L. Li, Q. Peng, J. X. Yi, X. Wang, and Y. Li, "Near monodisperse $\mathrm{TiO}_{2}$ nanoparticles and nanorods," Chemistry-A European Journal, vol. 12, no. 8, pp. 2383-2391, 2006. 
[16] C.-S. Kim, B. K. Moon, J.-H. Park, B.-C. Choi, and H.-J. Seo, "Solvothermal synthesis of nanocrystalline $\mathrm{TiO}_{2}$ in toluene with surfactant," Journal of Crystal Growth, vol. 257, no. 3-4, pp. 309315,2003

[17] J. M. Wu, "Low-temperature preparation of titania nanorods through direct oxidation of titanium with hydrogen peroxide," Journal of Crystal Growth, vol. 269, no. 2-4, pp. 347-355, 2004.

[18] J.-M. Wu, S. Hayakawa, K. Tsuru, and A. Osaka, "Nanocrystalline titania made from interactions of $\mathrm{Ti}$ with hydrogen peroxide solutions containing tantalum chloride," Crystal Growth and Design, vol. 2, no. 2, pp. 147-149, 2002.

[19] X. Peng and A. Chen, "Aligned $\mathrm{TiO}_{2}$ nanorod arrays synthesized by oxidizing titanium with acetone," Journal of Materials Chemistry, vol. 14, no. 16, pp. 2542-2548, 2004.

[20] O. K. Varghese, D. Gong, M. Paulose, K. G. Ong, E. C. Dickey, and C. A. Grimes, "Extreme changes in the electrical resistance of titania nanotubes with hydrogen exposure," Advanced Materials, vol. 15, no. 7-8, pp. 624-627, 2003.

[21] T. Kudo, Y. Sasaki, M. Hashimoto, and K. Matsumoto, "Oxalato complexes directly formed by the reaction of interstitial carbides with hydrogen peroxide," Inorganica Chimica Acta, vol. 145, no. 2, pp. 205-209, 1988.

[22] Y. Yagi, M. Hibino, and T. Kudo, "Evaluation of titanium dioxide (anatase) from oxalato-polytitanate as an active material for rechargeable lithium batteries," Journal of the Electrochemical Society, vol. 144, no. 12, pp. 4208-4212, 1997.

[23] S. Ahmad, B. Jousseaume, T. Toupance et al., "A new route towards nanoporous $\mathrm{TiO}_{2}$ as powders or thin films from the thermal treatment of titanium-based hybrid materials," Dalton Transactions, vol. 41, no. 1, pp. 292-299, 2012. 

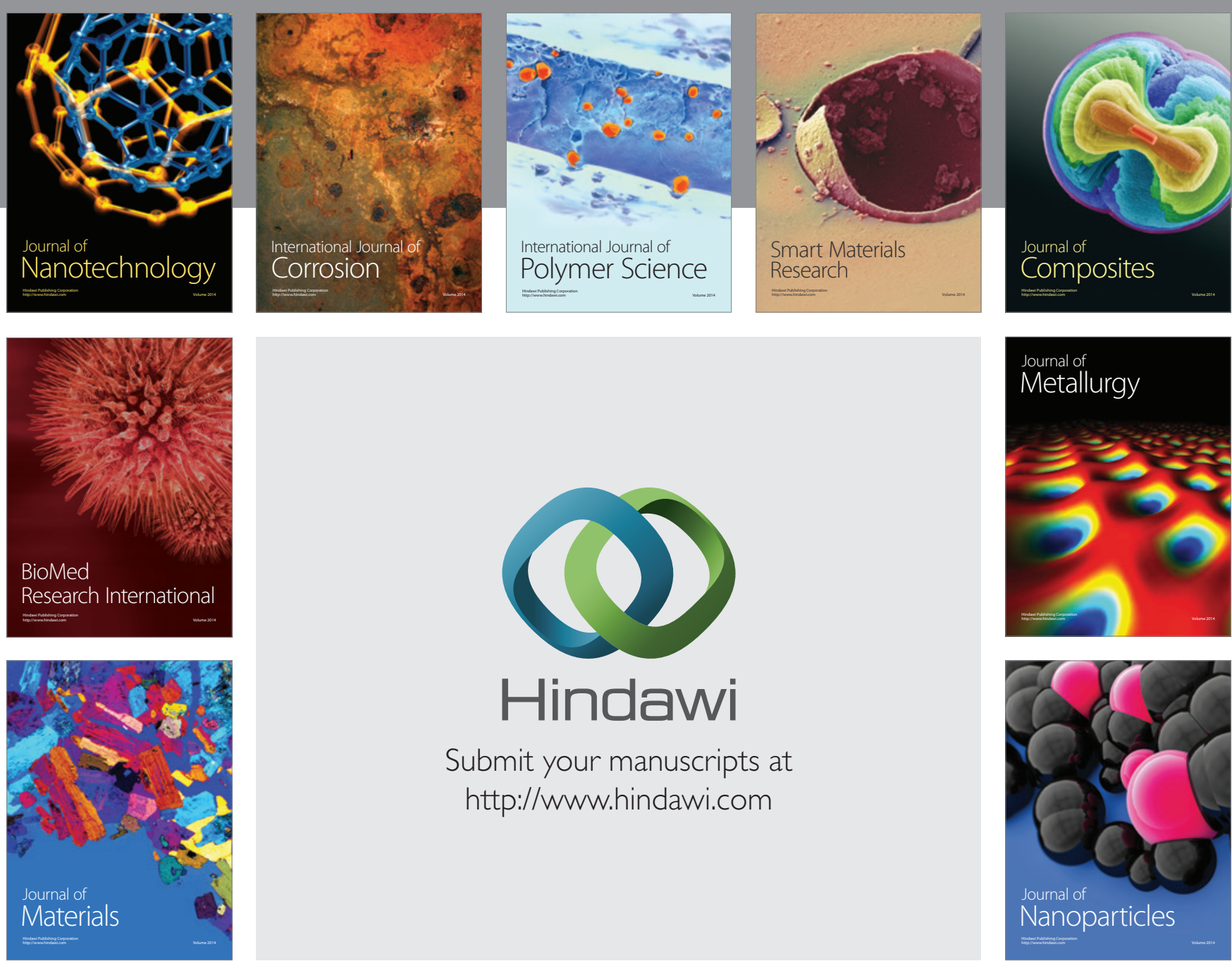

\section{Hindawi}

Submit your manuscripts at

http://www.hindawi.com



Materials Science and Engineering
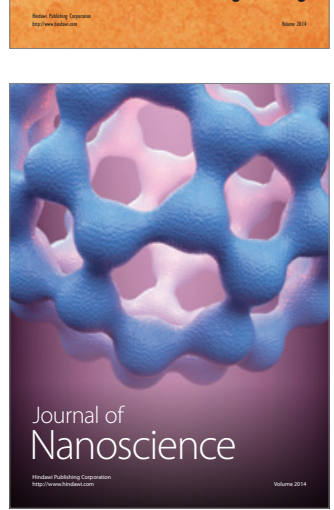
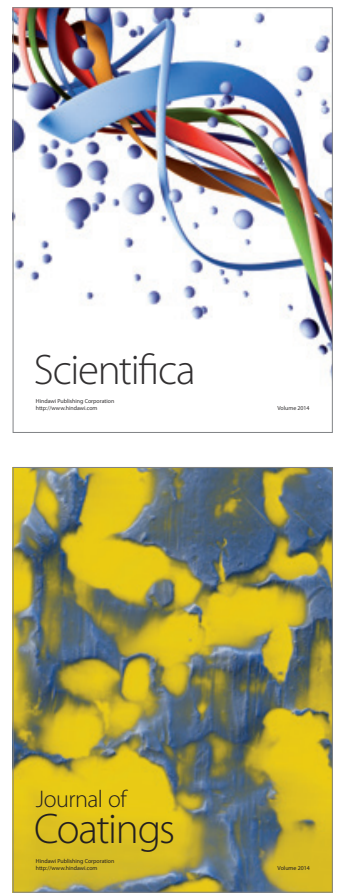
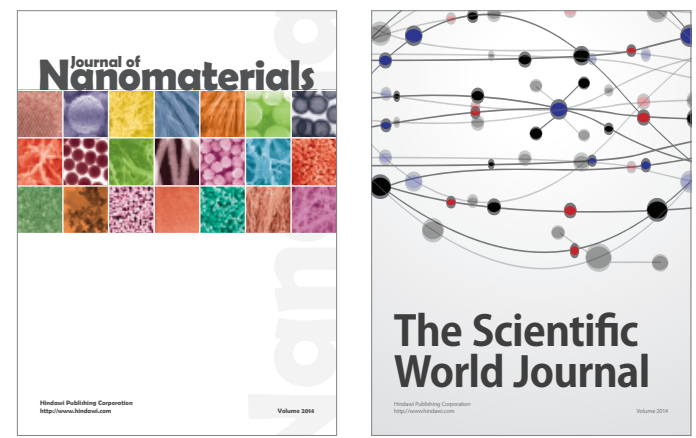

The Scientific World Journal
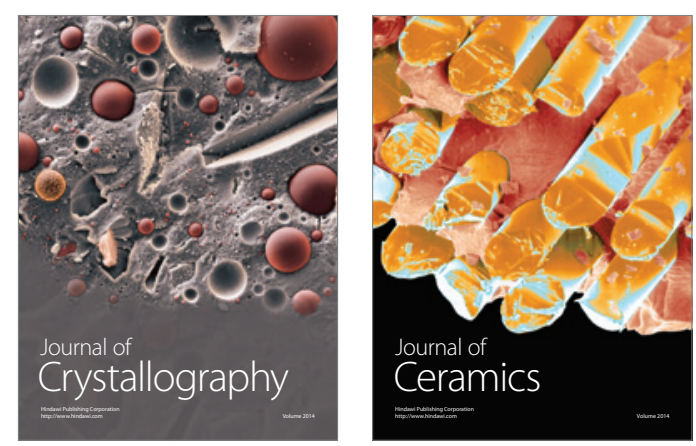
